\title{
NeuroFAST - the Integrated Neurobiology of Food Intake, Addiction and Stress
}

\author{
Julian G. Mercer Sue P. Bird \\ Rowett Institute of Nutrition and Health, University of Aberdeen, Aberdeen, UK
}

This special edition of OBESITY FACTS contains a series of review papers based around components and themes of the NeuroFAST research programme, which is funded by the European Union Seventh Framework programme (FP7-KBBE-2009-3; grant agreement 245009). The NeuroFAST project was constructed in response to the published call text entitled 'Stress, Addiction and Eating Behaviour', which reflected the common ground in terms of neurochemical pathways and brain regions between eating behaviour, including eating disorders, and addiction biology. The project was designed to ask the question 'Does food addiction exist?'. The growing obesity epidemic is driven by over-consumption of calories, and evidence strongly implicates processes beyond homeostatic energy balance. Accordingly, addiction to food, or more frequently specific food items such as chocolate, is often touted in the popular press as a potential cause of over-consumption and weight gain. This possibility is also the subject of debate within the scientific literature where several lines of evidence substantiate similarities in mechanism, molecule, anatomy, and clinical and behavioural characteristics between over-eating and chemical addiction. For example, functional brain imaging studies in obese and addicted individuals have shown similar activation patterns when subjects are exposed to palatable food or drugs of abuse. However, the validity of the concept of food addiction has not been conclusively proven, and a more solid evidence base is required in this controversial area.

The aim of this special issue is to produce a focussed collection of reviews that will be of interest to a range of specialties within the broader scientific community, and that will also aid the NeuroFAST consortium in the generation of targeted research briefs. These are intended to provide European public health policy makers and other stakeholders with digestible scientific information to support future decision making. The contributions to the special issue cover the rewarding properties of food, the molecular and neuroanatomical components involved in food reward and addiction, and how stressors are integrated into 
these processes. The collected papers, authored by NeuroFAST scientists, draw information from both pre-clinical and clinical studies to assess the current evidential status of food addiction and how this compares, firstly, to the medically recognised forms of addiction that categorise substance use disorders as specific conditions with distinct definitions and diagnostic features and, secondly, to emerging concepts of behavioural addiction. For example, the term food addiction is mainly used in the context of industrially refined, 'highly palatable', multi-component, foods, such as sweetened beverages or high-fat diets, rather than of single components, which are the basis of chemical addiction.

Obesity is in essence the result of consumption of calories in excess of homeostatic requirements. Palatable food readily stimulates relative caloric over-consumption in both humans and laboratory animals, an outcome related to food reward and possibly to addictionlike processes. Pandit et al. [1] review the effects of a range of obesogenic diets on feeding behaviour, leptin resistance and hypothalamic neuropeptide systems, and how the properties of the diet influence these parameters. These interactions could be important in dietary reformulation to limit over-consumption and weight gain. Pandit et al. [1] argue that by understanding how and why the composition of the diet affects its reward value and addictive potential, it may be possible to develop novel foods that limit consumption and weight gain. The relationships between food properties and their sensory and physiological effects are discussed. The ability of different solid or liquid palatable diets or supplements to induce obesity and influence leptin signalling is discussed, before the authors proceed to consider effects on the brain reward circuitry. The neurobiological accompaniments to sucrose addiction are compared to those recorded from animals exposed to drugs of abuse, including the involvement of the endogenous opioid circuitry, which is dealt with in more detail by Noguieras et al. [2].

Many of the neural structures involved in addictive behaviour are also involved in food reward. Opioids (including endorphins, enkephalins, dynorphins and endomorphins) play an important role in neural reward processes that lead to addictive behaviour, both homeostatic and hedonic feeding mechanisms involve opioid neuropeptide systems, and opioid antagonists inhibit consumption of both addictive drugs and palatable food. These relationships suggest the existence of a form of opioid-related addiction focussed on palatable foods. Nogueiras et al. [2] review the pharmacological and endogenous evidence of opioid receptor involvement in energy balance, including their interactions with the melanocortin, neuropeptide $Y$ and orexin systems, reward processes and diet-induced obesity, and the relative potency of these effects, including clinical trial evidence where there is weight loss potential in obese patients. There are three main families of opioid receptors ( $\mu-, \kappa-$ and $\delta$-) of which $\mu$-receptors are most strongly implicated in reward and induce feeding even in satiated animals when injected into the NAcc. Likely connections with other components of the NeuroFAST research programme are implied by the evidence of involvement of opioids in eating disorders such as anorexia nervosa and in stress-induced eating. The use of genetically manipulated mice has strengthened understanding of the role of opioids and their receptors in energy balance, diet-induced obesity, glucose and lipid metabolism, and hedonic feeding. However, a number of avenues are identified for future study to resolve important gaps in our knowledge of the molecular mechanisms through which opioids influence the hedonic properties of food, including brain site-specific manipulation of gene expression and gender differences in biological effects.

The contribution of Menzies et al. [3] addresses the decision to eat and the processes that curtail feeding as well as the integration and balance of control of energy homeostasis, reward and stress in this outcome. Stress may influence the balance between the homeostatic and reward circuitry in the brain and increase food intake and preference for palatable foods. The authors suggest that the stress and reward pathways may be more tractable as 
drug targets than the homeostatic systems where redundancy and the impact of molecular substrates on multiple physiological systems may limit efficacy and suitability. Stressors of different types have a variety of effects on feeding behaviour, while the reward value of a palatable food can be sufficient to negate the affect of an aversive stimulus, and the authors review the evidence for involvement of the key peripheral hormones, leptin and ghrelin, in these interactions. There is also increasing evidence of involvement of the hypothalamic peptides, vasopressin and oxytocin, in the linkage between stress, reward and appetite. The authors hypothesise that these peptides affect the sensitivity of hypothalamic homeostatic neurons to systemic feedback signals such as leptin and ghrelin.

De Jong et al. [4] focus on behavioural and neurobiological similarities between binge eating disorder (BED), which is prevalent within the obese human population, and drug addiction, drawing upon evidence from human subjects and, in particular, from animal models. They argue that if food possesses addictive properties then food-addicted rats should exhibit similar behavioural responses when seeking and consuming food as they do when addicted to drugs, and that patterns of feeding may be diagnostic of susceptibility to food addiction. Accordingly, several models from the drug addiction field are assessed for application in the study of food addiction. Three criteria have been proposed for addictionlike behaviour in animals, based on the DSM-IV criteria for substance dependence. These are high motivation to seek the drug, difficulty limiting drug intake and continuation of drug seeking despite aversive consequences. A number of similar strands of evidence support addiction-like behaviour directed at food in animal models. Significantly, there is considerable variability in susceptibility to addictive behaviour in both animals and humans, and for food addiction-like behaviour the diet and type of reward may be important. Withdrawal behaviours and enhanced motivation are observed in restricted access diets, manipulations which may have some parallel in BED in humans.

Albayrak et al. [5] consider the existence of food addiction from the perspective of the medically established psychiatric classifications of substance abuse and in the light of the preliminary revision of the Diagnostic and Statistical Manual for Mental Disorders (DSM V; American Psychiatric Association) which might allow diagnosis of behavioural addictions. This proposed revision suggests replacing the previous category 'Substance-Related Disorders' with 'Addiction and Related Disorders', thus for the first time allowing the diagnosis of behavioural addictions. Although there are a number of reasons behind the reluctance of the medical profession to adopt this realignment, it is supported by the broad overlap in psychological characteristics between so-called 'chemical' and 'behavioural' addiction and the common properties of addictive substances or activities. These are reviewed in this contribution, along with the diagnostic validity of food addiction. An integration of over-eating into substance use disorders would imply a form of chemical addiction for which an evidence base does not exist, whereas there is evidence to suggest that food addiction might be viewed as a specific form of behavioural addiction in a subgroup of obese individuals. It is almost certainly inappropriate to invoke food addiction in the gradual accumulation of excess weight gain over prolonged periods of time that characterises the development of overweight and obesity in the majority of the population so affected. In contrast to such marginal over-eating, clinically significant and regular over-eating i.e. abnormal eating behaviour (currently classified as eating disorders) warrants consideration as food addiction. However, although eating disorders such as BED have characteristics that more closely resemble addiction, they do not always lead to obesity. Rodent models of binge eating also suggest the recruitment of neurobiological processes in over-consumption of food similar to those seen in addiction, and these may provide a link between chemical and behavioural addiction. More research into eating patterns, over-eating and their neurobiology is required to substantiate the potential categorisation as behavioural addiction. 
Positron emission tomography (PET) data has made a significant contribution to our understanding of the role of the brain in the control of food intake and obesity in humans. Iozzo et al. [6] review the evidence of regional brain activation induced by palatable food and the likely involvement of these brain regions in ingestive behaviours. Although similar activation patterns are reported in drug abuse and non-chemical addictions, the authors argue that such resemblances should be interpreted with caution. They review our current understanding of the changes that accompany obesity and meal feeding, and the involvement of dopamine receptors. For example, obese subjects display differences in the brain circuits related to palatability and reward, with evidence of chronic activation of somatosensory brain areas, reduced activity in cortical areas concerned with perceived reward and anticipated pleasure, differential response to food ingestion and reduced striatal D2 receptor availability. Changes in D2 receptor availability and cortical metabolism are also observed with substance addiction and may precede changes in behaviour. The authors conclude that at present there is insufficient data to conclude that common mechanisms determine emotional food dependency and addiction but that detailed psychological evaluation of subjects in order to distinguish different compulsive and addictive features could be productive.

The impact of stress on eating behaviour, and implications for obesity, introduced by Menzies et al. [3] are brought into the context of everyday life by Scott and Johnstone [7], who address stress in the workplace, including during shift working. The evidence suggests that stress affects health both directly via autonomic and neuroendocrine processes and indirectly through changes to health behaviours. For example, stress, or 'daily hassles', may be associated with increased snacking, a likely contributory factor to our obesogenic environment. Shift workers are at greater risk of a number of non-communicable diseases; they report a shift in the type of food consumed and, in particular, an increase in the proportion of energy-dense 'convenience' food consumed as well as an alteration in the distribution of energy intake throughout the day. The involvement of stress, and stressor type, in these relationships remains to be established. The direction of change in calorie intake due to stress appears to depend upon differences between individuals in eating behaviour and personality. Priorities for future research are reviewed, such as the need to apply more quantitative methods in free-living subjects, to differentiate the effects of workplace stress from that of daily life, to assess the benefits of healthy working environment initiatives and to investigate the effects of shift working in occupational groups other than offshore workers or medical staff.

\section{Conclusion}

To establish whether food addiction is 'fact or fiction' requires further investigation of the behavioural and neurobiological similarities between addiction and over-eating (in particular BED). The development of the evidence base in this area will involve both the study of appropriate volunteer and patient groups, and animal models from the drug addiction and feeding fields. High-calorie diets, similar to those consumed in Western society, influence brain reward centres and can generate molecular and behavioural changes similar to drugs of abuse, but the identity of the key ingredients in these diets is less certain. Disintegrating these complex diets to examine how specific components modulate the brain reward circuitry could have major implications for the battle against over-consumption of calories, informing the design of novel foods which are palatable yet fail to produce obesogenic, and addictive, consequences, or identifying neurobiological targets for pharmacological manipulation. Improved knowledge of the impact of stress, and in particular daily 
stressors, on our interaction with palatable foods should help shape life-styles and environments for the future. Readers are invited to keep up to date with progress in the NeuroFAST project by visiting the website ( $w w w$.neurofast.eu/), or by requesting inclusion on the project stakeholder list - please contact Julian Mercer (J.Mercer@abdn.ac.uk) or Sue Bird (Sue. Bird@abdn.ac.uk) for details.

\section{Acknowledgements}

The authors have received funding from the European Union Seventh Framework Programme (FP7/2007-2013) under grant agreement $n^{\circ} 245009$ (NeuroFAST).

\section{References}

1 Pandit R, Mercer JG, Overduin J, la Fleur SE, Adan RAH: Dietary factors affect food reward and motivation to eat. Obes Facts 2012;5:221-243.

2 Nogueiras R, Romero A, Vazquez MJ, Garrido M, López M, Diéguez C: The Opioid system and food intake: homeostatic and hedonic mechanisms. Obes Facts 2012;5:196-207.

3 Menzies JRW, Skibicka KP, Dickson SL, Leng G: Neural substrates underly ing interactions between appetite, stress and reward. Obes Facts 2012;5:208-220.

4 De Jong JW, Vanderschuren LJMJ, Adan RAH: Towards an animal model of food addiction. Obes Facts 2012; 5:180-195.

5 Albayrak Ö, Wölfle SM, Hebebrand J: Does food addiction exist? A phenomenological discussion based on the psychiatric classification of substance related disorders and addiction. Obes Facts 2012;5:165-179.

6 Iozzo P, Guiducci L, Guzzardi MA, Pagotto U: Brain PET imaging in obesity and food addiction: current evidence and hypothesis. Obes Facts 2012;5:155-164.

7 Scott C, Johnstone AM: Stress and eating behaviour: implications for obesity. Obes Facts 2012;5:277-287. 